\title{
Impulse Bursts of Geomagnetic Pulsations as the Ground Signature of Contact of Interplanetary Irregularity with the Dayside Magnetopause
}

V. A. Parkhomov', B. A. Dovbnya'2, N. L. Borodkova3 , A. V. Dmitriev4,5, V. V. Safargaleyev6, A. Y. Pashinin 7

\author{
${ }^{1}$ Baikal State University, Irkutsk, Russia \\ ${ }^{2}$ Geophysical Observatory Borok, Institute of Earth Physics RAS, Moscow, Russia \\ ${ }^{3}$ Institute of Space Research RAS, Moscow, Russia \\ ${ }^{4}$ Institute of Space Science, National Central University, Taiwan \\ ${ }^{5}$ Skobeltsyn Institute of Nuclear Physics Moscow State University, Moscow, Russia \\ ${ }^{6}$ Polar Geophysical Institute, Apatity, Russia \\ ${ }^{7}$ Institute of Solar-Terrestrial Physics Sibirean Branch RAS, Irkutsk, Russia \\ Email: pekines_41@mail.ru
}

Received 19 February 2016; accepted 4 March 2016; published 9 March 2016

Copyright (C) 2016 by authors and OALib.

This work is licensed under the Creative Commons Attribution International License (CC BY). http://creativecommons.org/licenses/by/4.0/

(c) (i) Open Access

\begin{abstract}
The aim of the present article was to analyze features of interactions between an interplanetary shock wave and the magnetosphere on the basis of comparative analysis of geomagnetic variations observed during a sudden commencement (SC) by satellites and ground-based stations with high time resolution. A case study of the interaction of an interplanetary shock wave (ISW) with the magnetosphere was carried out on April 23, 2012. Statistical analysis was based on 11 SC events occurring from 1988 to 2012 . A precursor of SC in the form of a broadband electro-magnetic pulse is detected globally in the frequency range of 0.2 to $6.5 \mathrm{~Hz}$. The spectrum of pulses has a resonant structure. It is assumed that the structure results from a filtering effect of the ionosphere on MHD waves generated at the front of the ISW or in its interaction with the magnetopause.
\end{abstract}

\section{Keywords}

Precursor of Sudden Commencement, Broadband Electromagnetic Pulse, Interplanetary Shock Wave-Related Waves, Ionospheric Alfven Resonator

Subject Areas: Geophysics

How to cite this paper: Parkhomov, V.A., Dovbnya, B.A., Borodkova, N.L., Dmitriev, A.V., Safargaleyev, V.V. and Pashinin, A.Y. (2016) Impulse Bursts of Geomagnetic Pulsations as the Ground Signature of Contact of Interplanetary Irregularity with the Dayside Magnetopause. Open Access Library Journal, 3: e2493. http://dx.doi.org/10.4236/oalib.1102493 


\section{Introduction}

One direction for investigation of the influence of the interplanetary medium on space weather is analysis of magnetospheric responses to interactions with solar wind inhomogeneities and plasma discontinuities. At the front of a discontinuity, an abrupt change occurs in solar wind parameters such as plasma density, velocity, temperature, and the magnitude and direction of the interplanetary magnetic field (IMF). In particular, a magnetohydrodynamic (MHD) disturbance resulting from the interaction can be used as a tool for diagnosis of intramagnetospheric plasma (so-called magneto-seismology, see, for example [1]).

A sharp jump in solar wind parameters permits easy association of magnetospheric disturbances with solar wind variations because the time moment of the discontinuity interaction with the magnetopause (hereafter $T_{0}$ ) can be determined unambiguously. As a rule, satellite data are used to determine the time propagation of an interplanetary front from an upstream monitor to the subsolar point of the bow shock. Unfortunately, this method has an uncertainty of several minutes because the interaction with the magnetopause is preceded by propagation of a solar wind structure through the magnetosheath, where the speed and configuration of the structure can change dramatically. In this case, ground-based observation can be an additional or even alternative method of $T_{0}$ determination.

A number of techniques are used for determination of $T_{0}$, such as monitoring of wave processes in a wide frequency range (geomagnetic field variations, pulsations, VLF emissions) and effects of precipitating particles (auroral absorption of space radio noise, auroras and ionospheric disturbances). The present study uses the geomagnetic response of the magnetosphere to interaction with an interplanetary shock wave (ISW).

In describing geomagnetic data (standard magnetograms), sudden commencement of magnetic storms is abbreviated as SSC and sudden impulse is abbreviated as SI. In addition, an asterisk in SSC* and SI* denotes the presence of a sharp decrease in the horizontal component of the geomagnetic field right before a sharp increase. A preliminary reverse impulse is abbreviated as PRI, and a sharp increase or a major impact is denoted as main impulse (MI) [2].

Variations in the geomagnetic field recorded during SSC are a combination of fields produced by various current systems arising at sharp perturbations of the magnetosphere, which is reflected in magnetograms in the form of variations with positive or negative signs. During SSC, various kinds of geomagnetic pulsations are excited in a wide frequency range from Pc1 to Pc5 with the nomenclature Psc1 to Psc5 [3]. Activation of Pc1 oscillations after SSC and SI is described in detail by Kangas et al. [4]. Safargaleev et al. [5] show that PRI is associated with an increase in the flux of precipitating particles, which is initiated when an ISW hits the magnetopause and generates a burst of wide-band geomagnetic pulsations in the frequency range $0.1 \mathrm{~Hz}$ to $2 \mathrm{~Hz}$.

Parkhomov [6] [7] finds a fine structure of PRI in the form of specific packets of oscillations with a discrete spectrum in the frequency range $0.1 \mathrm{~Hz}$ to $2 \mathrm{~Hz}$ and with a duration equal to the duration of PRI. These packets are called "the oscillatory structure of the preliminary impulse".

D'Costa and Dovbnya [8] showed that right after interaction of an ISW with the magnetosphere, Pc1 geomagnetic pulsations ( $\sim .2$ to $0.6 \mathrm{~Hz}$ ) with a sharp front were excited in the polar cup. Guglielmi [9] suggested that such pulsations were created by the interaction of the magnetosphere with a spatially periodic oscillation structure at the front of an oblique shock wave. Parkhomov et al. [10] analyzed the propagation of unstructured Pc1 pulsations. They established that the sharp leading edge of the pulsations was similar to that shown by D'Costa and Dovbnya (1974) during an SSC that was associated with compression of the magnetosphere and with a change in the frequency of emitters due to changes in the geomagnetic field strength in the region of development of cyclotron instability.

It is also important to note that interplanetary shock waves can also have an oscillatory structure, which is observed in situ by satellites [11] [12]. Therefore, a review of the literature shows that there are still inconsistencies in both observations and interpretations as well as the proposed mechanisms.

The present article is devoted to analysis of the interaction of an ISW with the magnetosphere on the base of satellite and ground-based observations with high temporal resolution and the possibility of determining interrelated effects. First, we analyze features of contact by an ISW with the magnetosphere on April 23, 2012. We then present statistical data on 11 SSC* events for the period 1988 to 2012.

\section{Observations and Analysis}

An SSC* event on April 23, 2012 was caused by interaction of the magnetosphere with a solar wind inhomo- 
geneity that was identified as an ISW. The analysis is based on experimental data on parameters of the solar wind and IMF acquired from 11 satellites (see Table 1). The GSM coordinates of the satellites are shown in Figure 1. According to OMNI data (http://cdaweb.cdaweb.gsfc.nasa.gov/), the distance to the subsolar bow shock was 13.02 Re at 0323 UT and it moved to 11.88 Re at 0324 UT.

The statistical analysis was based on observation data for the solar wind and IMF acquired from the Wind, ACE, Geotail (GTL) and IMP-8 satellites with a sampling rate of $0.017 \mathrm{~Hz}$ to $0.012 \mathrm{~Hz}$. The geomagnetic pulsations were recorded on the Russian network of observatories and the CARISMA network

(http://www.carisma.ca) by highly sensitive induction coil magnetometers with a sampling rate of 20, 40 and 64 Hz. The list of stations is shown in Table 2. Data from Intermagnet, the global network of magnetic observatories, were also used (http://intermagnet.org).

Preliminary analysis of the initial digital data was carried out using the spectral-temporal Fourier analysis [14]. The spectral-temporal analysis of the records was made using the SpektraPro program [15].

Table 1. Orbital coordinates and time of solar wind passage.

\begin{tabular}{ccccc}
\hline Satellite & Data & Xgse & Ygse & Zgse \\
\hline WIND & $02.15 .09 \mathrm{UT}$ & 260.1531 .9021 .54 & & \\
SOHO & $02.20 .00 \mathrm{UT}$ & 200.4054 .1813 .25 & & 1.47 \\
ACE & $02.26 .28 \mathrm{UT}$ & $223.94-17.50-12.41$ & 24.56 & 0.84 \\
THC & $03.06 .21 \mathrm{UT}$ & 59.87 & 24.43 & 7.75 \\
THB & $03.06 .31 \mathrm{UT}$ & 59.04 & 23.83 & -25.63 \\
GTL & $03.20 .06 \mathrm{UT}$ & 12.14 & -24.62 & -25.14 \\
C2 & $03.20 .28 \mathrm{UT}$ & 9.89 & -25.15 \\
C1 & $03.20 .29 \mathrm{UT}$ & 9.85 & 30.65 & -10.89 \\
C3 & $03.20 .35 \mathrm{UT}$ & 9.45 & 9.46 & \\
C4 & $03.20 .35 \mathrm{UT}$ & 0.33 & & \\
S_R & $03.20 .50 \mathrm{UT}$ & &
\end{tabular}

Table 2. Observatory locations and types of magnetometers.

\begin{tabular}{cccc}
\hline Observatory (Abbreviation) & Latitude geodet. & Longitude geodet. & Type of magnetometer \\
\hline Barentsburg (BRB) & $70^{\circ} .20$ & $15^{\circ} .82$ & Induction Coil \\
Lovozero (LOZ) & $67^{\circ} .97$ & $35^{\circ} .08$ & Induction Coil, Fluxgate \\
Borok (BOR) & $58^{\circ} .03$ & $38^{\circ} .33$ & Induction Coil \\
Uzur (UZR) & $52^{\circ} .17$ & $104^{\circ} .45$ & Induction Coil, Fluxgate \\
Mondy (MND) & $51^{\circ} .6$ & $100^{\circ} .9$ & Induction Coil. \\
Dawson (DAWS) & $64^{\circ} .05$ & $220^{\circ} .89$ & Induction Coil, Fluxgate \\
Fort Smith (FSMI) & $60^{\circ} .02$ & $248^{\circ} .05$ & Induction Coil, Fluxgate \\
Fort Churchill (FCHU) & $58^{\circ} .76$ & $265^{\circ} .92$ & Induction Coil, Fluxgate \\
Pinawa (PINA) & $50^{\circ} .20$ & $263^{\circ} .96$ & Induction Coil, Fluxgate \\
Barrow (BRW) & $71^{\circ} .32$ & $203^{\circ} .38$ & Fluxgate
\end{tabular}




\subsection{Case Event of ISW Interaction with the Magnetosphere}

On April 23, 2012, an ISW was observed at all 11 near-Earth satellites. It was detected by the following sequence of satellites: THB at 03:06:21.860 UT; THC at 03:06:37.725; Geotail at 03:20:06 UT; the Cluster satellites sequentially: C2-03:20:28 UT, C1-03:20:29 UT, C3-03:20:35, C4-03:20:35; and Spektr-R at 03:20:50 UT (Table 1 and Figure 1).

During the interaction of the ISW with the magnetosphere between 0300 and 0320 UT, the Cluster satellites were located upstream from the bow shock, as shown in Figure 1. The jump in solar wind parameters observed by Wind was as follows: $\Delta P=6.15 \mathrm{nPa}, \Delta N=19 \mathrm{~cm}^{-3}, \Delta V=66 \mathrm{~km} / \mathrm{s}, \Delta B=5 \mathrm{nT}$. It is important to note that the IMF was northward, and its average value was $B z=0.33 \mathrm{nT}$ for the 1-hour period prior to arrival of the ISW, and on the wave front the jump was positive: $\Delta B z=+1 \mathrm{nT}$. The jump in solar wind pressure caused motion of the bow shock and magnetopause toward earth. Various magnetopause models predict standoff distances varying from 8 Re to 9 Re for given solar wind conditions [16]. The model proposed by Lin et al. [17] was the most robust and advanced model; it gives a subsolar magnetopause distance of 8.26 Re for dynamic pressure $P d=9$ $\mathrm{nPa}$, IMF strength $B=9 \mathrm{nT}$, IMF $B z=6 \mathrm{nT}$ (observed by Wind) and an Earth dipole tilt angle of $p s i=0^{\circ}$ (equinox). The calculated bow shock and magnetopause positions (Figure 1) convince us that the Cluster satellites were in the solar wind during the entire time interval.

Contact of the ISW with the magnetosphere caused a global SSC. Stations in the auroral zone recorded an SSC* with PRI. The greatest SSC* amplitude was observed in the afternoon sector at the Barrow Intermagnet station (BRW) at $0321 \mathrm{UT}$ (1521 MLT): amplitude of PRI $A_{P R I}=171 \mathrm{nT}$ and amplitude of main pulse $A_{M I}=282$ $\mathrm{nT}$. The $T_{0}$ at this observatory was calculated as suggested by [1], $T_{0}=03: 21: 00 \pm 00: 30 \mathrm{UT}$. If $T_{0}$ is determined by the difference between the values of the $H$ component at 03:20:00 and 03:21:00, then $T_{0}=03: 20: 30$ UT (see Figure 2(a)).

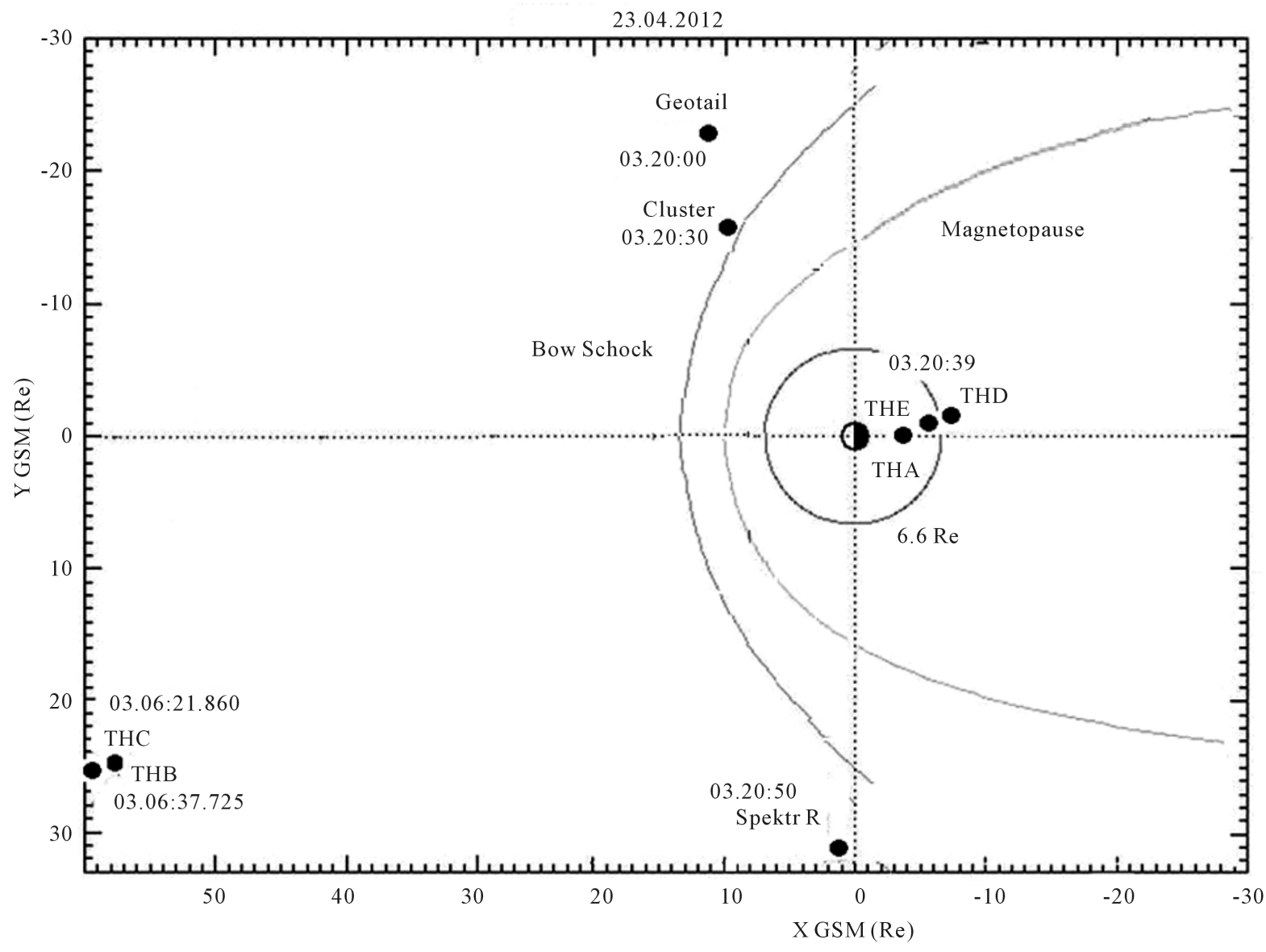

Figure 1. Positions of the satellites during the arrival of ISW 23.04.2012. 
(a)

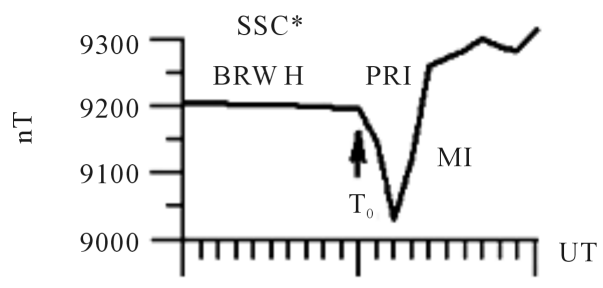

03.10:00 03.20:00 03.30:00



03.20:00

03.21:00 UT



03.20:00

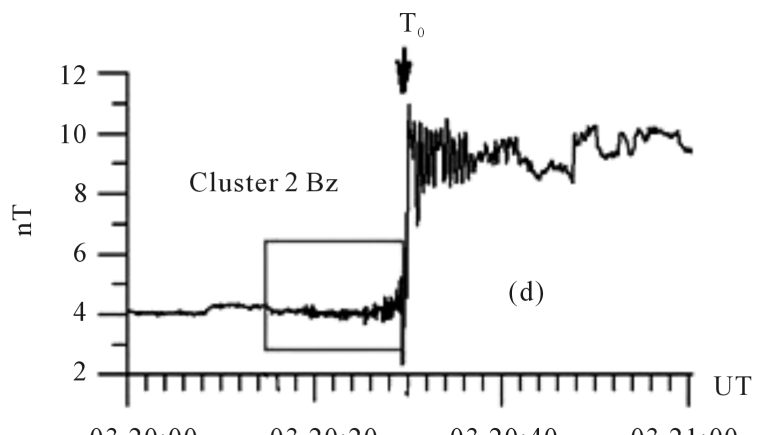

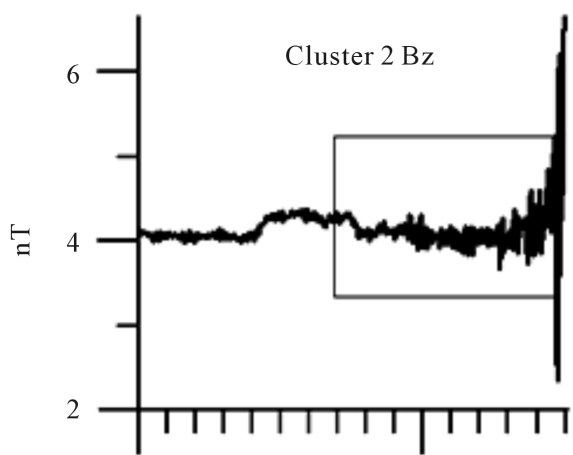

03.20:00

$03.20: 20$

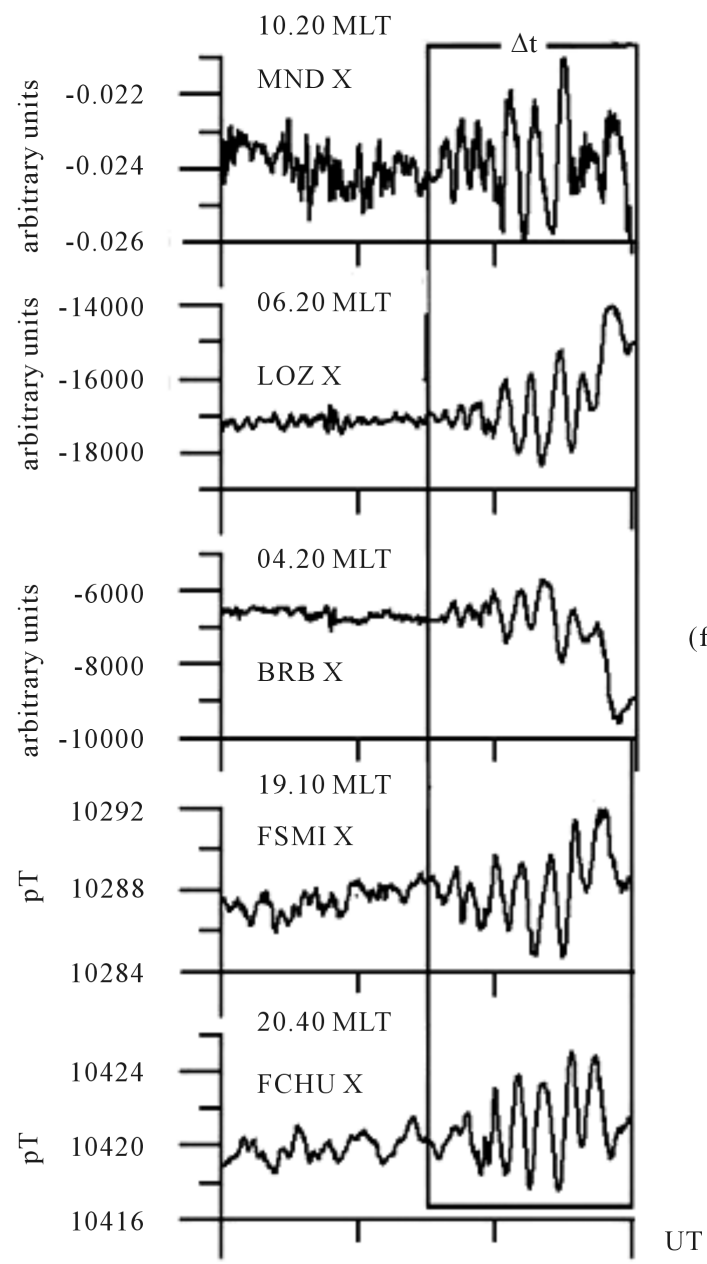

$03.20: 00$

Figure 2. Magnetospheric response to the contact with the ISW according to network coil induction and fluxgate magnetometers. (a) Fragment of magnetogram SSC* from BRW. X-is a component, PRI-preliminary recovery impulse, MImain impulse, $T_{0}$-beginning of magnetic field change. (b) Fragment of magnetogram of the X-component from the fluxgate magnetometer at FCHU observatory. The vertical arrows $T_{0}$ indicate the beginning of the SSC*. The rectangle is a precursor. (c) Fragment of magnetogram of the X-component from the coil induction magnetometer at FCHU observatory, showing a precursor SSC. The rectangle-a precursor. (d) Fragment of magnetogram IMF (Bz-component) obtained from the C2 Cluster 2 satellite that is near the magnetopause. The rectangle is a precursor or ISW oscillatory structure. (e) Fragment of magnetogram IMF (Bz-component) obtained on the satellite Cluster 2 which is near the magnetopause with more details. Rectangle is a precursor or ISW oscillatory structure. (f) Fragments of magnetograms from coil induction magnetometers on the network of observatories, showing a precursor SC. The rectangle is a precursor. 
We will define SSC* in according to simultaneous data of fluxgate and induction coil magnetometers. In Figure 2(b) one can see the FCHU-X is the fluxgate magnetometer at Fort Churchill observatory and $T_{0}$ is the beginning of ISW contact with the magnetopause. A portion of recorded component $\mathrm{X}$ of the induction coil magnetometer at the same observatory is shown in Figure 2(c); we can see a train of oscillations before $T_{0}$ with duration of $15 \mathrm{~s}$ (denote rectangular $\Delta t$ ). That train is represented in more detail in Figure 2(f) using magnetic data from two observatories of the CARISMA chain (the same train was detected at the Pinawa and Rabbit Lake stations) and at three stations in the Russian network. We see that modes of SSC* recording at the magnetometers were different. The records of the induction coil magnetometers at all observatories at 03:20:15 UT fixed the beginning of increasing amplitude of oscillations (A $\sim 5 \mathrm{nT}$ at the FCC observatory). Oscillations lasted $\sim 15$ seconds (indicated by a rectangle in Figure 2(c), Figure 2(f)).

In the records from the fluxgate magnetometers, deviation of the magnetic field component from undisturbed levels was detected at 03:20:30 UT (arrows in Figure 2(b)). This time coincides with the time of SSC* determined using a standard magnetogram at the Barrow observatory (Figure 2(a)). The data show a difference in $T_{0}$ depending on the type of device: The more sensitive induction coil magnetometers allow determination of effects that are invisible to the fluxgate magnetometers. The pulsation train was simultaneously detected by coil induction magnetometers while preserving the shape and period at observatories in longitude range of about $250^{\circ}$ and latitude range of $\sim 20^{\circ}$ (surrounded by the rectangle in Figure 2(f)).

Let's compare $T_{0}$ with the ISW arrival time at the C2 Cluster satellite, the one closest to the magnetopause. This point is considered the moment of ISW contact with the magnetosphere. The record of the IMF vertical component at Cluster C2 is presented in Figure 2(d) and in more detail at the top of Figure 2(e). It is clearly seen that the ground effect of the beginning measurement for the magnetic field by the fluxgate magnetometer (arrows in Figure 2(a), Figure 2(b)) coincides with the jump in IMF at the ISW (Figure 2(d), Figure 2(e)).

Therefore, in the beginning, 03:20:30 UT can be taken as the SSC* $\left(T_{0}\right)$. However, we emphasize that up to this point the train of oscillations had already been recorded (starting at 03:20:15 UT), which is readily identifiable in the recordings by induction coil magnetometers on both the day and night sides of Earth (denoted by the rectangle in Figure 2(c), Figure 2(f)). The beginning and duration of the train of oscillations coincided with the duration of the train of oscillations in the interplanetary magnetic field (oscillatory structure of the ISW), which was observed by Cluster C2 upstream of the bow shock.

Combining all the observations leads to two preliminary conclusions:

1) Determination of the start time $T_{0}$ for the magnetospheric response depends on the type of magnetometer.

2) If $T_{0}$ is taken as the start of geomagnetic field changes on fluxgate magnetometers, the train of oscillations recorded by induction coil magnetometers can be seen as a precursor of SSC*.

Figure 3 can serve as confirmation of the preliminary findings, which showed the IMF $(B z)$ measured by Cluster C2 (Figure 3(c)), the solar wind flux (nV) measured by Spektr-R (Figure 3(d)), and ground-based data from induction coil (component X) (Figure 3(b)) and fluxgate magnetometers (components H, D) (Figure 3(a)) at the Lovozero observatory (MLT $=0620$ ). In Figure 3 one can accurately determine the casting time SSC* (a vertical arrow on Figure 3(a), Figure 3(b)), which coincides with a jump from 4 to $10 \mathrm{nT}$ of the Bz component of IMF at the ISW front detected by Cluster C2 at 03:20:28 UT (the arrow in Figure 3(c)). The recording from the induction coil magnetometer showed a train of oscillations (indicated by a rectangle) with frequency range changes within $0.26 \pm 0.08 \mathrm{~s}$. The train was detected before the start of the preliminary impulse of sudden commencement, as can clearly be seen in component $\mathrm{D}$ as a decrease before a sharp increase. The oscillation train on Earth outran the magnetic field jump detected in front of the ISW by the Cluster satellites. It is noteworthy that the oscillation structure was detected by the Cluster satellites in all components of the IMF (oscillations of the $\mathrm{Bz}$ component are shown by a rectangle in Figure 3(c)). It is well known that waves with frequencies below 3 $\mathrm{Hz}$ are observed upstream of low Mach number (2 - 3) interplanetary shocks [11] [12]. The amplitude of oscillations increases towards the ISW front. The oscillation period varies in the range $\sim 2.1 \div 3.65$ ( $\left.f_{\text {average }} \sim 2.74\right) \mathrm{Hz}$ (Figure 3(e)). Note that at the ground stations, the frequencies of pulsations varied in the range $0.26 \pm 2.0 \mathrm{~Hz}$ with falling frequency (Figure 3(f)).

The Specktr-R satellite did not detect any oscillations in the solar wind flux (Figure 3(d)). A 20-second delay in the time that the ISW passed through Spektr-R relative to the Cluster satellites in terms of the moment of the IMF jump is of interest. This delay was probably related to separation of the satellites within $\sim 10 \mathrm{Re}$ in the $\mathrm{X}$ coordinate and a slightly inclined interplanetary shock wave front. 

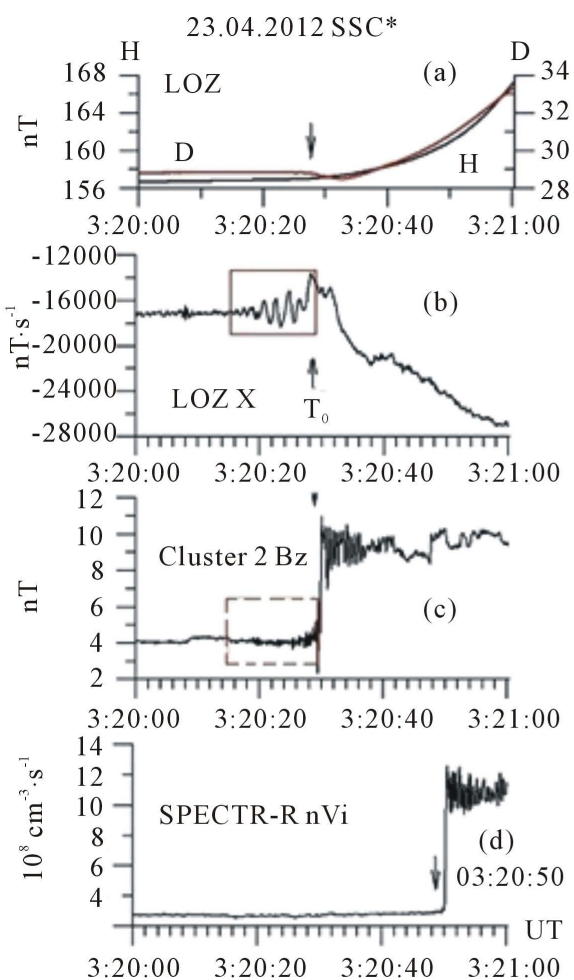

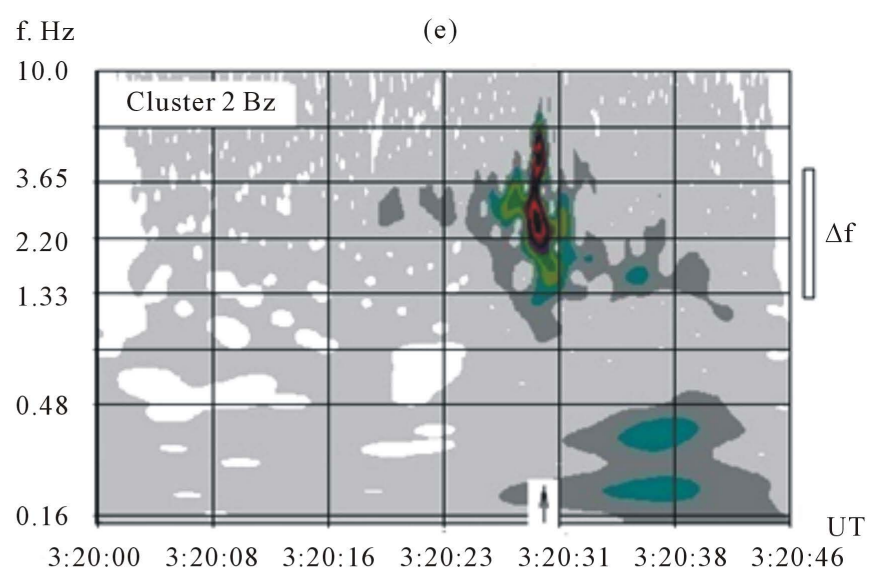

(f) $\quad \mathrm{T}_{0}$

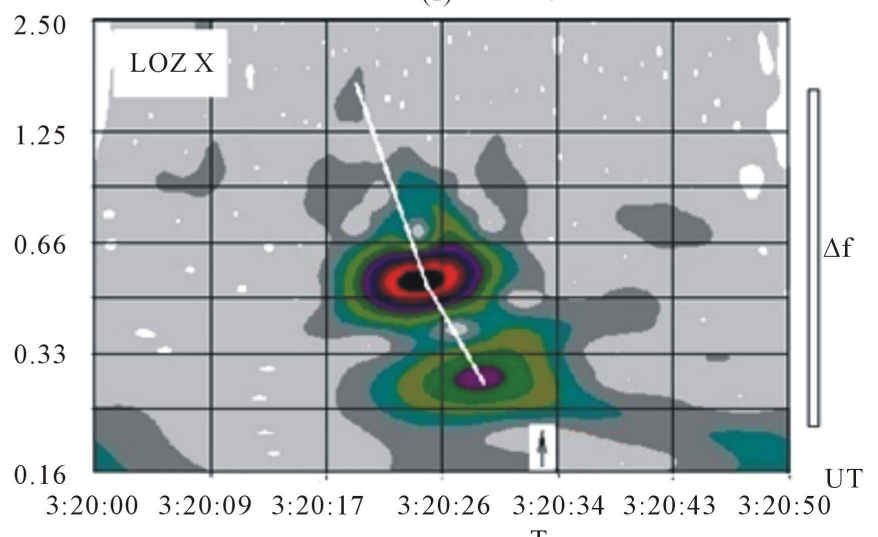

$\mathrm{S}(\mathrm{T})$ arbitrary units

$22 \quad 46 \quad 100 \quad 215464 \quad 1000 \quad 2154 \quad 4642$

Figure 3. Ground response to contact of the magnetosphere with ISW. (a) Fragment of fluxgate magnetogram from Lovozero observatory (H, D are components). (b) Fragment of the induction coil magnetogram ( $\mathrm{X}$ is a component) from the same observatory. (c) Fragment of the magnetogram IMF (Bz is a component) obtained from the C2 Cluster satellite that is near the magnetopause. (d) Variations in the solar wind flux registered at the Spectrum R satellite. (e) Dynamic spectrum of oscillations $\mathrm{Bz}$ IMF measured by $\mathrm{C} 2$ Cluster 2 satellite. $\Delta f$-difference between maximum and minimum frequency in the burst. (f) Dynamic spectrum of oscillations X component measured by coil induction magnetometer at the Lovozero observatory.

Let's consider the spectrum properties of the precursor. In the spectrograms obtained from analysis of the taped records of induction coil magnetometers with the help of the SpectraPro program (Figure 4), the oscillation train (the precursor) looked like a broadband outburst (denoted by the white arrows) in the range of periods of $0.5 \div 3.8 \mathrm{~s}(0.26 \div 2.0 \mathrm{~Hz})$. The outburst was identical in all observatories in longitude range $\sim 250^{\circ}$ and latitude range $\sim 20^{\circ}$. The spectra in all observatories had a common feature - a discrete structure. The discrete nature was revealed in strengthening and weakening of the spectral signal power in certain frequency ranges. In that case, the maximum distribution of power in the spectrum were fixed in ranges of 1.75, 0.81, 0.53, and 0.29 $\mathrm{Hz}$. We will call this structure of energy distribution in the spectrum "similar resonance" and named SRSspectral resonance structure [18]. The main spectral maximum was observed in the range $0.55 \pm 0.66 \mathrm{~Hz}$ at all observatories from low latitudes up to the polar cap.

The resonance structure of the spectrum for the network of stations can also be traced in the spectrograms obtained using spectral-temporal analysis (see Figure 5). The maximum spectral frequency fell in frequencies of $1.75,0.81,0.53$, and $0.29 \mathrm{~Hz}$. Another important feature of the spectrum was simultaneous decreases in the oscillation frequency at all observatories by the end of the event (a white broken line in Figure 5). These spectrum properties could indicate a common source of the oscillation mode in the space-distribution observatories. 

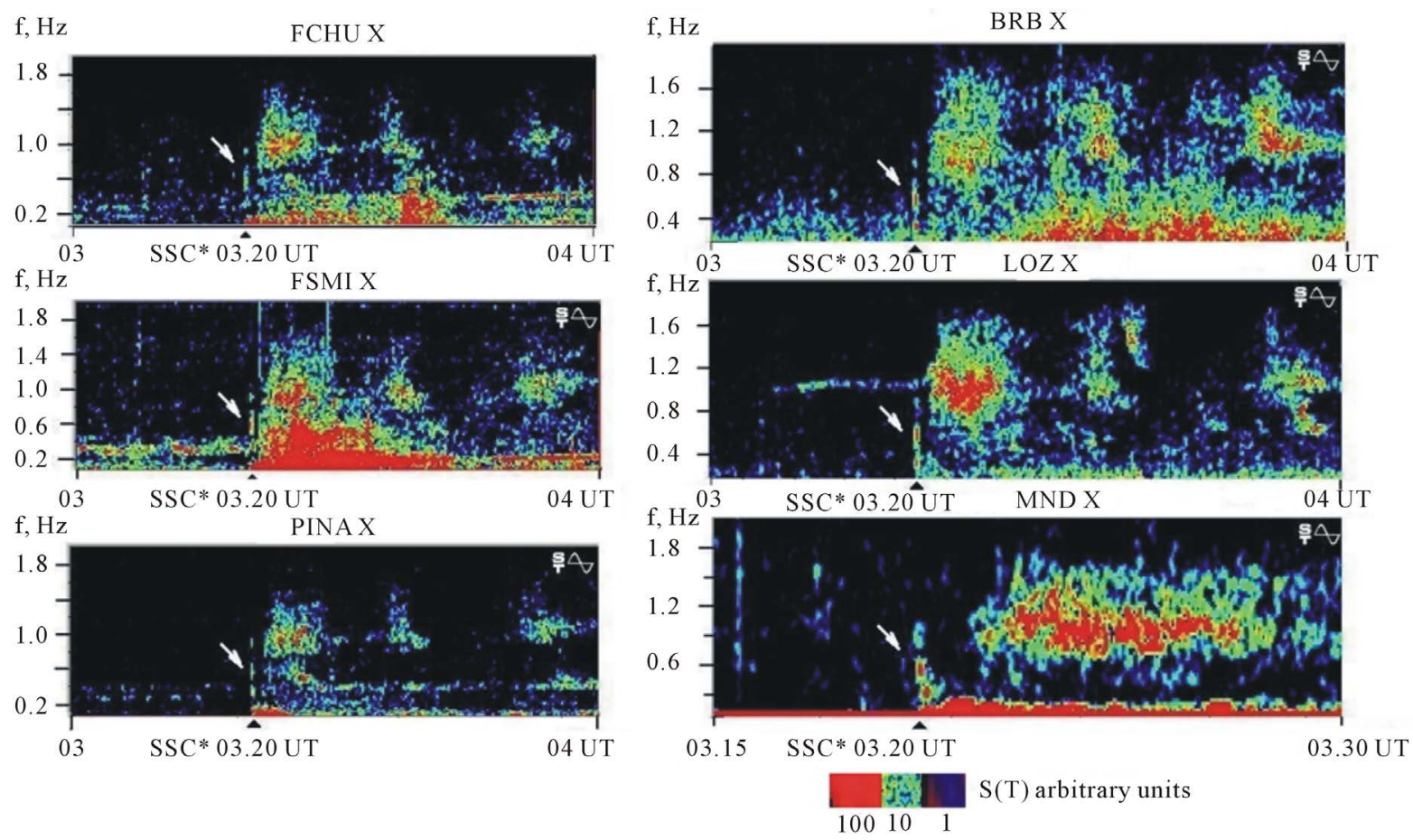

Figure 4. Fragments of sonograms of geomagnetic pulsations from the observatoryi network. The arrows indicate the broadband impulse with the resonant structure of the spectrum. Oscillograms of the impulse are shown in Figure 2(f) (closed by a rectangle).

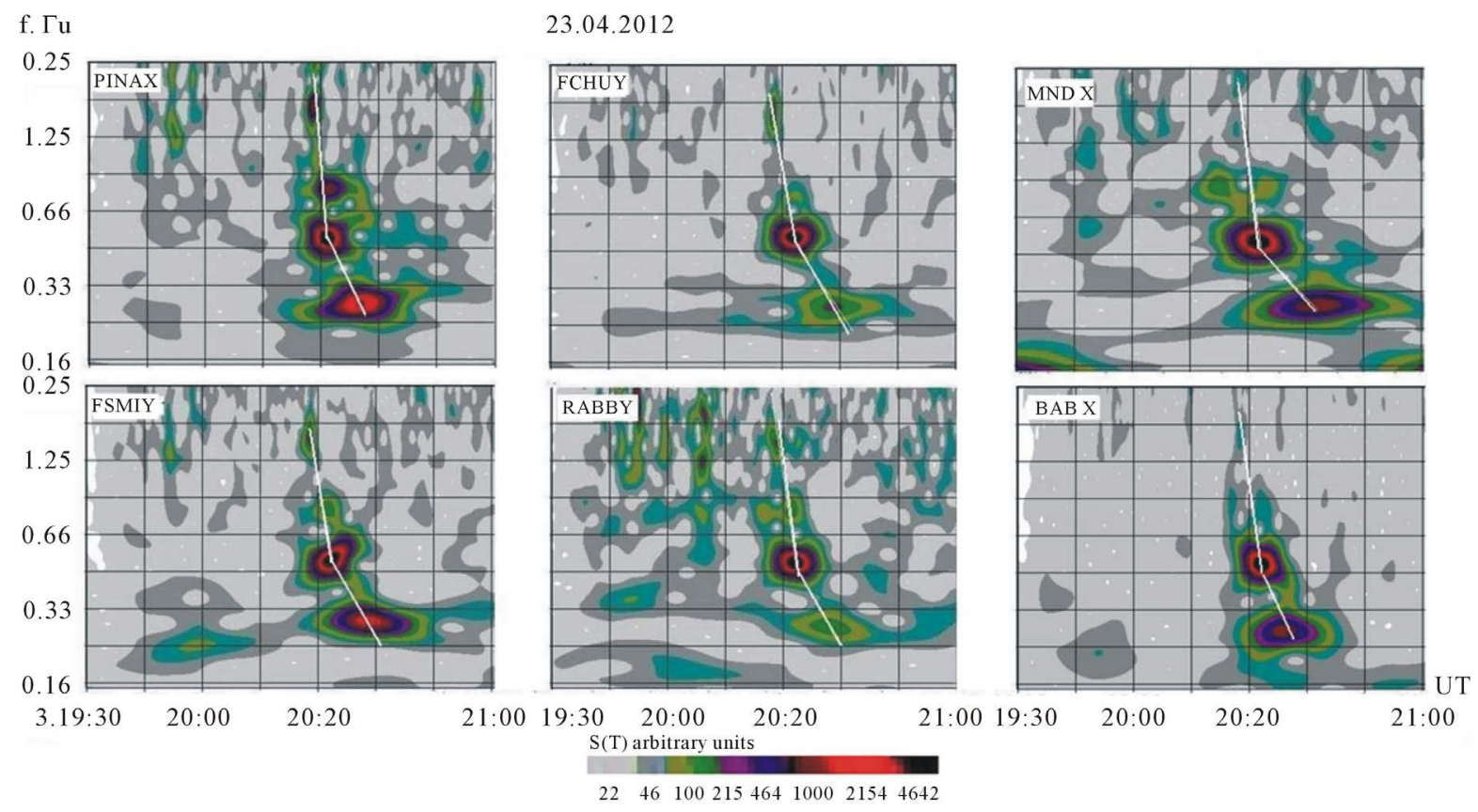

Figure 5. Pictures of the dynamic spectrums of broadband pulses indicated by the arrows in Figure 4.

Thus, on the basis of the data, it can be concluded that in the event considered, contact of the sharp front of the ISW with the oscillatory structure in front of it generated a train of damped oscillations with durations of 15 s. The amplitude of the oscillations in the train increased and the frequency decreased by the beginning of the actual SSC* generation. The oscillation amplitude recorded by observatories on the night side reached $\sim 5 \mathrm{nT}$. 
As for dayside observatories, there was no available data for the amplitude frequency characteristics of induction coil magnetometers, so the oscillation amplitudes in Figure 2 and Figure 3 are given in arbitrary units. The spectrum of oscillations in the train had a resonance structure at all observatories, and that data was used. The distribution of energy in the spectrum contained four spectral maxima at frequencies of $1.75,0.81,0.53$, and $0.29 \mathrm{~Hz}$. Simultaneous observations that the oscillation train showed a similar spectral structure at the network of stations confirmed that the phenomenon observed was not random, and that it preceded the beginning $\left(T_{0}\right)$ of SSC* on the Earth's surface. Let's call this a broadband impulse (oscillation train) as a precursor of SSC*.

The precursor was detected globally as an oscillation train up to 30 seconds in duration with amplitude increasing by the end of the train. The spectrograms showed the precursor to be like a broadband impulse of falling frequency (outburst) within the range of $0.2 \div 2 \mathrm{~Hz}$ with spectral resonant structure (SRS).

\subsection{Results of Statistical Analysis}

The statistical analysis used experimantal data on geomagnetic pulsations measured by the induction coil magnetometer of the Borok observatory and recorded on an analogue tape recorder. The analysis was based on a number of sudden changes in $\mathrm{H}$-component corresponding to the preliminary and main impulses. The width of frequency range of the pulses was determined. The spectral-temporal analysis of the records was performed using SpectraPro® software. We also used information about solar wind conditions at fronts of ISW. The key numbers used in the statistical analysis are listed in Table 3.

Non-randomness of the observed phenomenon was confirmed by statistics on broadband outburst observations detected during sudden commencements with giant amplitudes in the auroral zone from 1988 to 2012 at the Borok observatory. Some of the statistical data is presented in Figure 6 in the form of spectrograms of outburst fragments of magnetograms of auroral observatories during the hours when the observatory was in the afternoon sector. First of all, Figure 6 shows that in all cases, the broadband impulses with a resonant spectral structure were accompanied by sudden commencement with large amplitudes of the preliminary impulse (average value $\Delta H_{\text {pri }}=40.5 \mathrm{nT}$ ) and the main impulse SSC (average value $\Delta H_{s c}=219.9 \mathrm{nT}$ ) (see Table 3). It is important to note that all cases of broadband outbursts with discrete spectral structures were observed at large jumps in solar wind density and velocity under northward IMF and at positive jumps in IMF $\mathrm{Bz}$ at the front of the ISW.

The last column of Table 3 gives the range of frequencies occupied by the precursor. The range of changing $\Delta f$ is equal to $0.24 \mathrm{~Hz}$ to $6.5 \mathrm{~Hz}$. In Table 3, most of the events (8) of outbursts with frequency bursts range in the interval $\Delta f 0.24 \mathrm{~Hz}$ to $3.5 \mathrm{~Hz}$. These frequencies correspond to the frequency band of the ionospheric Alfven resonator (IAR) of $0.5 \mathrm{~Hz}$ to $3 \mathrm{~Hz}$ [18] [19] and over the IAR of $0.15 \mathrm{~Hz}$ to $0.3 \mathrm{~Hz}$ [20] [21].

\begin{tabular}{|c|c|c|c|c|c|c|c|c|}
\hline Дата & Время UT & $\Delta H_{p r i} \mathrm{nT}$ & $\Delta H_{s s c} \mathrm{nT}$ & $\Delta B_{I M F} \mathrm{nT}$ & $\Delta B z_{I M F} \mathrm{nT}$ & $\Delta \mathrm{V} \mathrm{km} / \mathrm{s}$ & $\Delta \mathrm{N} \mathrm{sm}^{-3}$ & $\Delta f \Gamma ц$ \\
\hline 07.12.1988 & 18.24 & - & 441 & - & - & - & - & 6.2 \\
\hline 20.01.1989 & 12.32 & 15 & 35 & - & - & - & - & 3.5 \\
\hline 13.03.1989 & 01.27 & 12 & 64 & - & - & - & - & 6.1 \\
\hline 16.03.1989 & 05.32 & 48 & 78 & 17 & +16 & 220 & 8 & 6.5 \\
\hline 29.12.1989 & 06.54 & 40 & 129 & 15 & +10 & 120 & 12 & 2.6 \\
\hline 04.06.1991 & 15.36 & 10 & 65 & 12 & +10 & 150 & 12 & 1.1 \\
\hline 08.06.1989 & 19.53 & - & 137 & 16 & +2 & - & - & 0.8 \\
\hline 20.03 .1990 & 22.43 & 29 & 368 & 14 & +18 & 100 & 27 & 0.24 \\
\hline 23.04.1991 & 10.41 & 171 & 282 & - & - & - & - & 0.7 \\
\hline 06.04.2000 & 16.30 & 100 & 400 & 18 & +2 & 195 & 12 & 0.4 \\
\hline 24.01 .12 & 15.03 & 20 & 100 & 19 & +16 & 160 & 9 & 0.3 \\
\hline Average & & 52 & 196 & 16 & +14 & 158 & 10 & 2.35 \\
\hline
\end{tabular}

$\Delta H, \Delta B_{I M F}, \Delta \mathrm{Bz}_{\mathrm{IMF}}, \Delta \mathrm{V}, \Delta \mathrm{N}$-jump in solar wind and IMF parameters at the front of the interplanetary shock wave. 
(a)
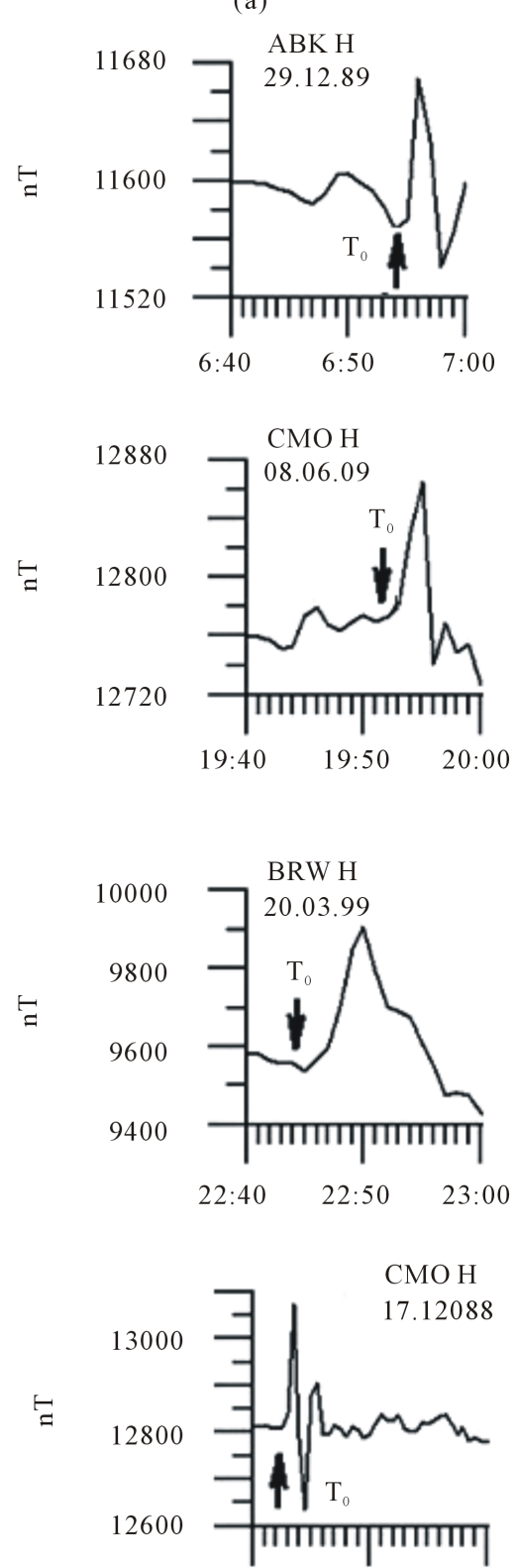

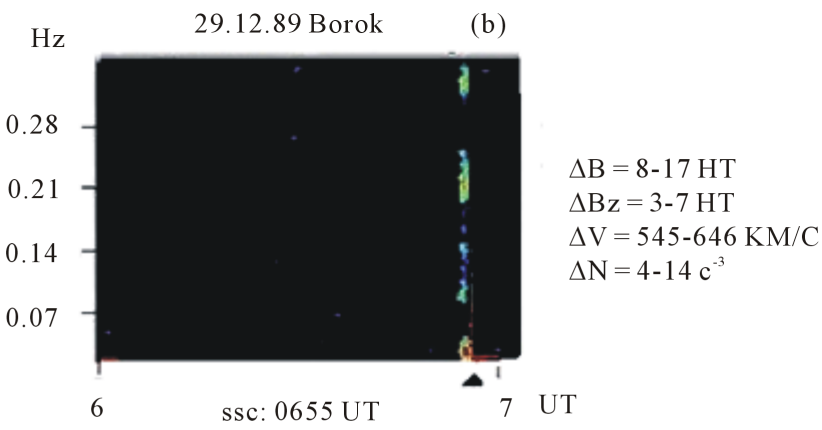

$\mathrm{Hz} \quad 08.06 .09$ Borok

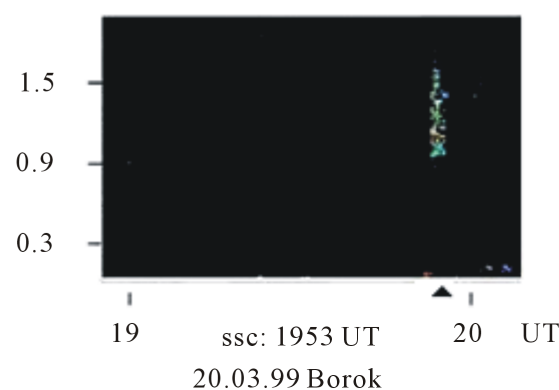

$\Delta \mathrm{B}=21-32 \mathrm{HT}$

$\Delta \mathrm{Bz}=1.2-4 \mathrm{HT}$

$\Delta \mathrm{V}=600-800 \mathrm{KM} / \mathrm{C}$

$\Delta \mathrm{N}=3-12 \mathrm{c}^{-3}$

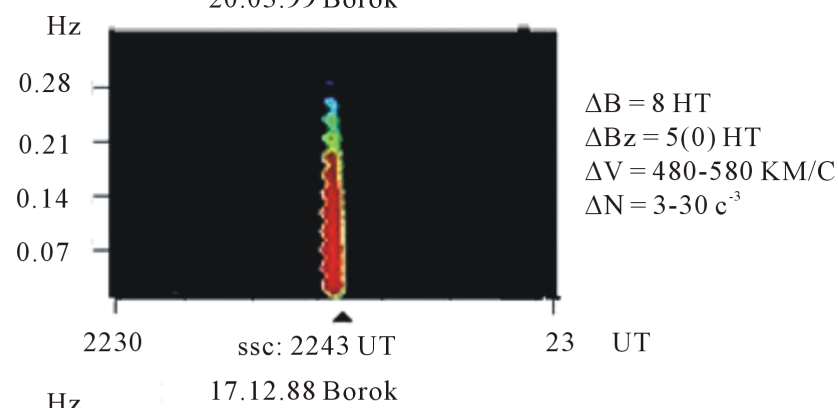

Figure 6. (a) Fragments of magnetograms from the auroral observatories, taken at the time of registration of SSC on the dayside of the magnetosphere; (b) Examples of broadband bursts - precursors SC.

\section{Discussion}

Mishin et al. [22] reported geomagnetic pulsations with a resonant spectral structure in the frequency range of $0.2 \mathrm{~Hz} \div 0.02 \mathrm{~Hz}$ that were excited by switching a gutter unsteadiness on the magnetopause during sharp compression and expansion of the magnetosphere when a magnetic cloud was passing Earth on January 10-11, 1997. Safargaleev [23] mentioned a similar response of the geomagnetic field (a short-term broadband outburst on sonograms) to a pressure jump in the solar wind. However, that study did not discuss the morphological properties or nature of the outburst.

Only the characteristics of precursors of sudden commencement have been studied to date; there have been no reliable interpretations of the effect. There are three possibilities: 
1) Interplanetary shock waves with large, sharp increases in solar wind pressure at the front result in a gigantic SSC followed by broadband outbursts of geomagnetic pulsations in the frequency range $0.2 \mathrm{~Hz} \div 6.5 \mathrm{~Hz}$. The outbursts have a resonant spectral structure. Note that a similar distribution of energy in the spectrum is characteristic of the ionospheric Alfven resonator, predicted theoretically and experimentally validated [18] [19]. According to those results, the first resonance frequency of the Alfven resonator varies between $0.5 \mathrm{~Hz} \div 3 \mathrm{~Hz}$ depending on the condition of the ionosphere; the energy distribution in the spectrum of electromagnetic noise increases at the values of the harmonics of the fundamental frequency of the resonator. However, it is likely that interpretation of the Pc1 wave precursor as an IAR response cannot be accepted. The IAR eigenfrequency depends strongly on latitude and local time, in contrast with the observed wave feature.

In addition to the IAR, the work of Dovbnya et al. [20] shows that Over Ionosperic Alvfen Resonator (OIAR) is associated with the IAR. It is manifested as discrete specific multiple frequencies above $0.15 \mathrm{~Hz} \div 0.3 \mathrm{~Hz}$ and fills the range of frequencies below the fundamental resonance of the IAR.

Let's compare our observations with the properties of the IAR and OIAR. First, in the event of April 23, 2012, the frequency range of the observed oscillation train fell within the range of the resonator frequencies (Figure 5). The number of harmonics in the spectrum varied by observatory and depended on latitude and components of the geomagnetic field. Thus, the harmonic of the $0.31 \mathrm{~Hz}$ frequency was absent from the Y component of the Mondy and Uzur observatories and from the $\mathrm{X}$ component of the Lovozero observatory. Let's also refer to the work of Dovbnya et al. [24], which analyzed the impact of MHD resonators on geomagnetic pulsations. This article gave examples of dynamic spectra of different types of geomagnetic pulsations and electromagnetic impulses observed on Earth during sudden storm commencements and earthquakes. The general nature of the dynamic spectra, which have a discrete character in the frequency band from $0.1 \mathrm{~Hz}$ to $6 \mathrm{~Hz}$, allows the assumption that appearance of discreteness in the pulsation spectrum is due to filtering of the MHD waves while they are passing through the ionosphere.

2) Another possible source of the broadband pulse could be direct transmission of a wave structure from the solar wind to the earth's surface. However, origin of differences between the spectral patterns of oscillations observed in the upstream solar wind and in the precursor recorded by the network of ground-based stations is not clear.

3) The small delay between the entries of the train observed at mid-night observatories and in the midday observatory corresponds to the results of SSC* pulse propagation during a storm on 24 March 1991 [22]. It was found a simultaneous worldwide start of the first impulse sudden onset. The authors suggested the presence of almost instantaneous propagation mode below the ionosphere. However, the physical nature of such regime was not discussed in the article [25].

\section{Conclusions}

1) It was found that a precursor appeared before the SSC*, caused by the interplanetary shock wave with a large, sharp increase in solar wind pressure at the front with a mostly positive IMF Bz. On April 23, 2012, the precursor was detected globally as a train of oscillations with frequency falling by the end of the event. The spectrum of the impulse had a resonant (discrete) structure with a duration of up to 30 seconds and amplitude increasing by the end of the train (up to $5 \mathrm{nT}$ ). On the spectrograms of geomagnetic pulsations associated with the gigantic SSC, the precursor looked like a broadband impulse (outburst) in the frequency range of $0.2 \mathrm{~Hz} \div$ $6.5 \mathrm{~Hz}$ of falling frequency with a resonant (discrete) spectral structure. Discreteness of the spectrum was strengthening of oscillation energy on some frequencies and weakening on others.

2) Statistical analysis of 14 events allowed determination of the frequency range of the impulses as $0.2 \mathrm{~Hz} \div$ $6.5 \mathrm{~Hz}$.

3) Only the characteristics of precursors of sudden commencements had been studied; there were yet no reliable interpretations of this effect. The broadband impulses generated by the interaction of interplanetary shock waves with the magnetosphere were the precursors of sudden commencements and could lead to mistakes in the determination of the $T_{0}$ of sudden commencements.

\section{Acknowledgements}

The data for ACE, IMP-8, Wind, Geotail, Cluster and THEMIS satellites obtained from the database of the NASA CDAWeb. The authors are grateful to the relevant PI for the opportunity to use these data. The authors 
are also grateful to the staff of the Russian Space Research Institute for the opportunity to use data from the Spektr-R satellite. The authors thank J. Mann, and D.K. Milling for access to the data from the CARISMA and CANOPUS networks, and the owners of the information for the opportunity to use global network data from the Kyoto World Data Center for Geomagnetism and the INTERMAGNET network. The data from the induction coil magnetometers at the Barentsburg and Lovozero observatories were made available by the Polar Geophysical Institute. The authors thank G.N. Zastenker for his constructive comments and interest in the work; B.I. Kline (Geophysical Observatory Borok) EPI for assistance in processing variations in the geomagnetic field that were placed on magnetic tapes; and A.V. Suvorova for calculations of positions of the magnetopause.

This work was partially supported by the Russian Foundation for Basic Research (RFBR grants 13-02-00819 and 10-02-01063) and grant NSC103-2923-M-006-002-MY3/ 4-05-92002HHC Taiwan-RFBR.

\section{References}

[1] Chi, P.J., Lee, D.-H. and Russel, C.T. (2006) Tamao Travel Time of Sudden Impulses and Its Relationships to Ionospheric Convections Vortices. Journal of Geophysical Research, 111, A08205. http://dx.doi.org/10.1029/2005JA011578

[2] Curto, J.J., Araki, T. and Alberca, L.F. (2007) Evolution of Concept of Sudden Storm Commencements and Their Operative Identification. Earth, Planets and Space, 59, i-xii. http://dx.doi.org/10.1186/BF03352059

[3] Nishida, A. (1980) Geomagnetic Diagnosis of the Magnetosphere. World, 222

[4] Kangas, J., Guglielmi, A. and Pokhotelov, O. (1998) Morphology and Physics of Short-Period Magnetic Pulsations. (Review). Space Science Reviews, 83, 435-512. http://dx.doi.org/10.1023/A:1005063911643

[5] Safargaleev, V.V.A., Kozlovsky, A.F., Honary, F.A., Voronin, A.T. and Turunen, T. (2010) Geomagnetic Disturbances on Ground Associated with Particle Precipitation during SC. Annals of Geophysics, 28, 247. http://dx.doi.org/10.5194/angeo-28-247-2010

[6] Parkhomov, V.A. (1985) On Fine Structure of Preliminary Impulse of Sudden Storm Commencement. Geomagnetism and Aeronomy, 26, 420-424.

[7] Parkhomov, V.A. (1990) The Oscillatory Structure of the Preliminary Impulse of Sudden Storm Commencement. Geomagnetism and Aeronomy, 30, 210-215.

[8] D’Costa, A. and Dovbnya, B.V. (1974) Sporadic Radiation in Polar Cap in Diapason 0.1-0.7 Hz. Geomagnetism and Aeronomy, 14, 1125.

[9] Guglielmi, A.V. (1979) MHD Waves in near Earth Plasma. M., Science, 108.

[10] Parkhomov, V.A, Dmitriev, A.V. and Tsegmed, B. (2015) On the Origin of Burst Pc1 Pulsations Produced in Interaction with an Oblique Interplanetary Shock. Planetary and Space Science, 109, 21-31. http://dx.doi.org/10.1016/j.pss.2014.12.008

[11] Russel, C.T. and Greenstadt, E.W. (1979) Initial ISEE Magnetometer Results: Shock Observations. Space Science Reviews, 23, 3-37.

[12] Tsurutani, B.T., Smith, E.J. and Jones, D.E. (1983) Waves Observed Upstream of Interplanetary Shocks. Journal of Geophysical Research, 88, 5645-5656. http://dx.doi.org/10.1029/JA088iA07p05645

[13] Zelyony, L.M., Zastenker, G.N., Petrukovich, A.A., Chesalin, L.S., Nazarov, V.N., Prokhorenko, V.I., et al. (2015) Plasma-F Experiment: Three Years of On-Orbit Operation. Solar System Research, 49, 580-603.

[14] Levshin, A.A., Frantsuzova, V.I. and Shkadinskaya, G.V. (1968) Spectral-Temporal Analysis of Seismic Wave. Computer Seismology M, 4, 197.

[15] http://www.vmiab.com/products/software/spectra-pro/

[16] Suvorova, A.V. and Dmitriev, A.V. (2015) Magnetopause Inflation under Radial IMF: Comparison of Models. Earth and Space Science, 2, 107-114. http://dx.doi.org/10.1002/2014EA000084

[17] Lin, R.L., Zhang, X.X., Liu, S.Q., Wang, Y.L. and Gong, J.C. (2010) A Three-Dimensional Asymmetric Magnetopause Model. Journal of Geophysical Research, 115, A04207. http://dx.doi.org/10.1029/2009ja014235

[18] Polyakov S.V. and Rapoport, V.O. (1981) Ionospheric Alfven Resonator. Geomagnetism and Aeronomy, 21, 610-614.

[19] Belyaev P.P., Polyakov, S.V., Rapoport, V.O. and Trakhtengerts, V.Y. (1989) Experimental Study of the Electromagnetic Background Noise in the Range of Short-Period Geomagnetic Pulsations. Izvestiya Vysshikh Uchebnykh Zavedenii, Radiofizika, 32, 663-672.

[20] Dovbnya, B.V., Guglielmi, A.V., Potapov, A.S. and Rakhmatulin, R.A. (2013) Additional Resonator for Ultra-Low Waves. Geophysical Research, 14, 49-58. 
[21] Dovbnya, B.V., Guglielmi, A.V., Potapov, A.S. and Kline, B.I. (2013) On the Existence of an over Ionospheric Alfven Resonator. Solar-Terrestrial Physics, 22, 12-15.

[22] Mishin, V.V., Parkhomov, V.A., Tabanakov, I.V. and Hayashi, K. (2001) About Switching Gutter Unsteadiness on Magnetopause during Passing the Earth a Magnetic Cloud on January 10-11, 1997. Geomagnetism and Aeronomy, 41, 165-168.

[23] Safargaleev, V.V. (2011) Perturbations of the Magnetosphere-Ionosphere System in the Arctic and the Problem of Space Weather Monitoring. PhD Thesis, Polar Geophysical Institute, Apatity.

[24] Dovbnya B.V., Guglielmi, A.V., Potapov, A.S. and Rakhmatulin, R.A. (2014) On Impact of MHD Resonators on Geomagnetic Pulsations. Geophysical Research, 6, 32-40.

[25] Araki, T., Fujitani, S., Emoto, M., Yumoto, K., Shiokawa, K., Ichinose, T., et al. (1997) Anomalous Sudden Commencement on March 24, 1991. Journal of Geophysical Research, 102, 14075-14086.

http://dx.doi.org/10.1029/96JA03637 